\title{
Energy-efficient RF Source Power Control for Opportunistic Distributed Sensing in Wireless Passive Sensor Networks
}

\author{
A. Ozan Bicen Ozgur B. Akan \\ Next-generation and Wireless Communications Laboratory \\ Department of Electrical and Electronics Engineering \\ Koc University, Istanbul 34450, Turkey \\ Email: \{abicen,akan\}@ku.edu.tr
}

\begin{abstract}
Energy limitation of sensor nodes is the main constraint to be addressed while designing and implementing algorithms for wireless sensor networks (WSN). Recently, to mitigate battery depletion problem and extend network lifetime, wireless passive sensor networks (WPSN) have become a new field of interest. Modulated backscattering is an important communication technique for WPSN to enable unlimited lifetime for sensor nodes. Determination of required number and power level of RF sources for wireless power transfer to sensor nodes is crucial for energy-efficient distributed sensing operation. Furthermore, deployed RF sources can share spectrum opportunistically via incorporation of cognitive radio capability such that desired distributed estimation distortion can be achieved with minimum spectrum utilization by WPSN. Employment of RF sources that radiate power only when spectrum opportunities are available unveils passive opportunistic distributed sensing (PODS).

In this paper, first, we model intercepted power by passive sensor from RF sources and reflected power by passive sensor at the sink, and effect of opportunistic access to licensed spectrum bands on instantaneous throughput of sensor nodes. Then, a power level control scheme for RF sources is proposed to achieve desired distortion level with minimum energy consumption while using opportunistic distributed sensing in WPSN. Achieved estimation distortion at sink with respect to number and power level of RF sources, and available spectrum opportunities is investigated, and energy saving provided by proposed power control scheme is assessed for various distortion requirements, channel noise levels, and available spectrum opportunities via simulation experiments.
\end{abstract}

Keywords-Wireless passive sensor networks, modulated backscattering, opportunistic spectrum access, estimation diversity.

\section{INTRODUCTION}

Collaborative sensing and resource-constrained nature are the main characteristics of the wireless sensor networks (WSN). Sensor nodes are deployed with tiny batteries because of size and price limitations, and maintenance of sensors is usually hard to realize due to random deployment. Therefore, wireless passive sensor networks (WPSN) [1] is introduced to overwhelm limited life-time problem of WSN via utilizing RF power radiated by external sources placed in the network.

RF sources can charge batteries or empower modulated backscattering of sensor nodes [1]. In order to provide DC

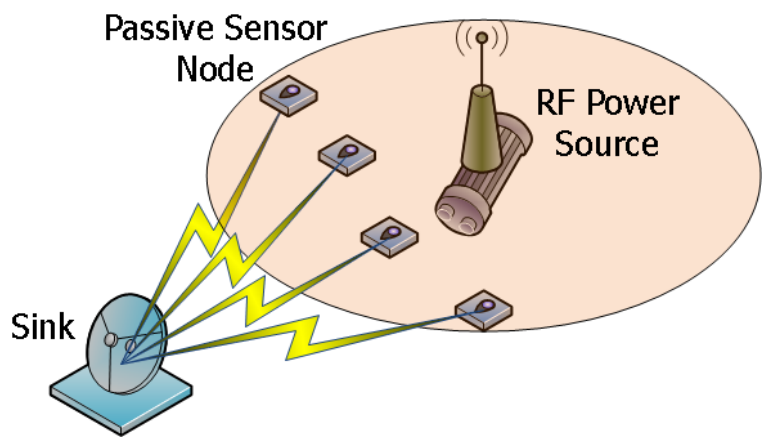

Figure 1. Remote powered passive sensor nodes communicating with sink via modulated backscattering.

power, RF-to-DC converter requires induction of $100 \mathrm{mV}$ on the antenna [2]. This power can be used to either operate sensor node or charge battery. WPSN nodes can use modulated backscattering communication technique, i.e., they can modulate the incident wave from RF source by changing its antenna impedance and reflect it [3]. When compared with other units' power consumption, the communication power consumption is the dominating factor for sensor nodes. Therefore, passive sensor nodes can help to resolve the lifetime constraint of traditional WSN. Backscattered electromagnetic wave by sensor nodes can be received at sink, and sinks can either collaborate in distributed estimation of event signal or forward the collected samples to a fusion center. Since event estimation is performed via collaboration of distributed passive sensor nodes, energy efficiency and estimation performance is closely coupled [5]. Passive sensor nodes powered by an external RF power source and using modulated backscattering for communication with sink is illustrated in Fig. 1.

Cognitive radio enables detection of unused licensed channels and opportunistic communication over them while they are unused [6]. Cognitive radio capable RF sources can detect spectrum holes for power radiating and energize passive sensor nodes over these detected vacant channels. Since modulated backscattering employing sensor nodes use the intercepted power from RF sources to transmit their 


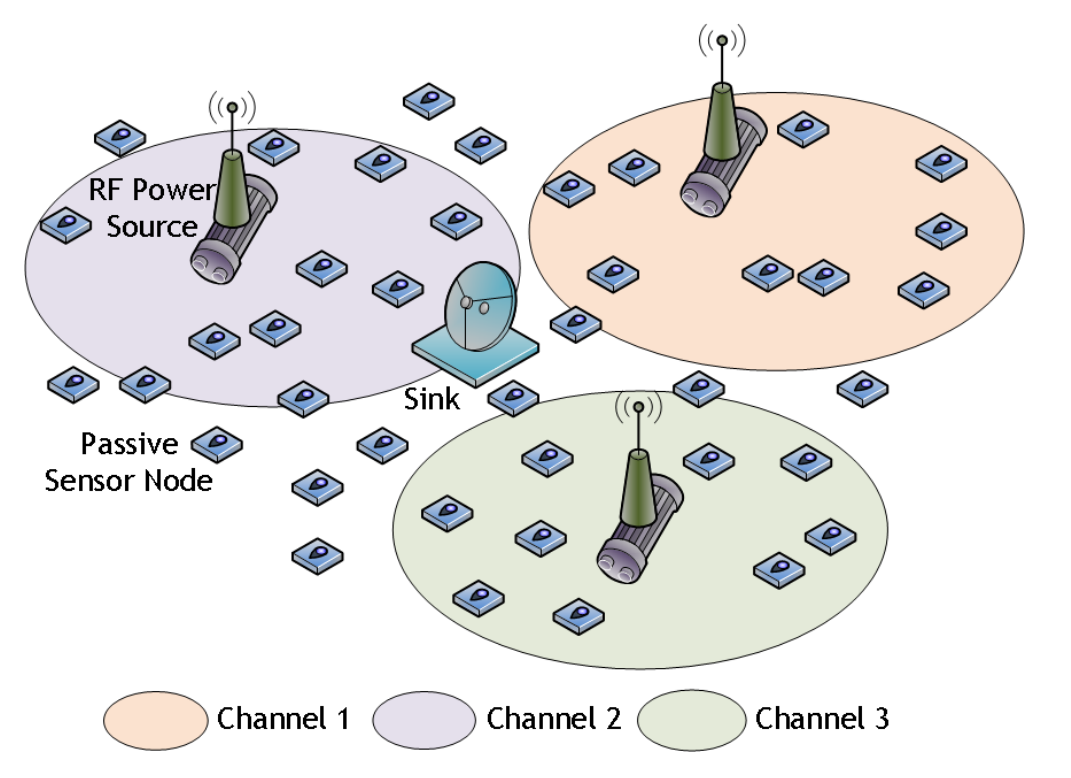

Figure 2. Passive opportunistic distributed sensing architecture.

observations, estimation distortion is directly effected by opportunities in the licensed spectrum as well as the number and power level of RF sources, i.e., RF source diversity. Passive sensor nodes powered by opportunistic spectrum access (OSA) capable RF power sources defines passive opportunistic distributed sensing (PODS). An example PODS topology is presented in Fig. 2.

In this paper, first, we devise an analytical model for estimation distortion which incorporates the number and power level of RF sources as well as instantaneous throughput limitation due to OSA. Using developed model, an RF source power control scheme is proposed to minimize energy consumption while satisfying estimation distortion requirements at the sink. Then, we investigate the effect of RF source diversity and available spectrum opportunities on estimation performance, and assess the energy saving provided by proposed scheme for various estimation requirements, channel noise levels, and spectrum opportunities via simulation experiments. Provided results in this work form a basis for the design of reliable and efficient communication techniques for opportunistic distributed sensing in WPSN.

The remainder of this paper is organized as follows. Passive opportunistic distributed sensing model is presented in Section II. An algorithm for energy-efficient RF power level adjustment while satisfying required estimation distortion is presented in Section III. Performance evaluations are presented in Section IV. Finally, the concluding remarks are given in Section V.

\section{Passive Opportunistic Distributed Sensing}

In this section, first, we model power transfer from RF sources to passive sensor nodes and reflected power by passive sensors at sink. Then, we detail used distributed estimation model for distortion calculations. Finally, we present OSA model and formulate instantaneous throughput due to OSA.

\section{A. Backscattered Power in WPSN}

Here, received power at sink node $m$ from passive sensor node $k$ is modelled. Log-distance shadowing propagation model [4] is used for backscattered power calculations. Path loss for intercepted power from RF source $l$ by sensor node $k\left(P_{k, l}^{\mathrm{I}}\right)$ can be calculated as

$$
\left(\frac{P_{k, l}^{\mathrm{I}}}{P_{l}^{\mathrm{RF}}}\right)_{\mathrm{dB}}=10 \log _{10} K_{k, l}^{\mathrm{I}}-10 \gamma \log _{10}\left(\frac{d_{k, l}}{d_{0}}\right)-\psi_{\mathrm{dB}}
$$

where $d_{0}$ is the reference distance, $d_{k, l}$ is the distance between sensor node $k$ and RF source $l, K_{k, l}^{\mathrm{I}}$ is the intercepted power from RF source $l$ by sensor node $k$ at distance $d_{0}$, $\gamma$ is the path loss exponent, and $\psi_{d B}$ is a Gaussian random variable with zero mean and $\sigma_{\psi_{d B}}^{2}$ variance. Reference path loss $K_{k, l}^{\mathrm{I}}$ for intercepted power by sensor node $k$ at distance $d_{0}$ from RF source $l$, can be calculated according to Friis propagation model [7] as $K_{k, l}^{\mathrm{I}}=\frac{P_{d 0}^{\mathrm{I}}}{P_{l}^{\mathrm{RF}}}=\left(\frac{G_{l}^{\mathrm{RF}}}{4 \pi d_{0}^{2}}\right) \cdot \sigma_{k}$, where $G_{l}^{\mathrm{RF}}$ is the antenna gain of RF source $l, \sigma_{k}$ is the radar cross section (RCS) area for sensor node $k$. Path loss for reflected power at sink $m$ by sensor node $k\left(\frac{P_{m, k}^{\mathrm{R}}}{P_{k, l}^{\mathrm{I}}}\right)$ can be calculated as

$$
\left(\frac{P_{m, k}^{\mathrm{R}}}{P_{k, l}^{\mathrm{I}}}\right)_{\mathrm{dB}}=10 \log _{10} K_{m, k}^{\mathrm{R}}-10 \gamma \log _{10}\left(\frac{d_{m, k}}{d_{0}}\right)-\psi_{\mathrm{dB}}
$$

where $K_{m, k}^{\mathrm{R}}=\frac{P_{d 0}^{\mathrm{R}}}{P_{k, l}^{1}}=\frac{G_{m}^{\mathrm{RF}}}{4 \pi d_{0}^{2}} \cdot A_{e m}$, and $A_{e m}$ is the antenna effective area for sink $m$, i.e., $A_{e m}=\frac{G_{m}^{\mathrm{RF}} \lambda_{k}^{2}}{4 \pi}$, for $\lambda_{k}$ is 


$$
\begin{aligned}
P_{m, k}^{\mathrm{R}} & =10 \log _{10} K_{m, k}^{\mathrm{R}}-10 \gamma \log _{10}\left(\frac{d_{m, k}}{d_{0}}\right)-\psi_{\mathrm{dB}}+\sum_{l=1}^{L} P_{k, l \mathrm{~dB}}^{\mathrm{I}} \\
& =10 \log _{10} K_{m, k}^{\mathrm{R}}-10 \gamma \log _{10}\left(\frac{d_{m, k}}{d_{0}}\right)-\psi_{\mathrm{dB}}+\sum_{l=1}^{L}\left(P_{l}^{\mathrm{RF}}{ }_{\mathrm{dBm}}+10 \log _{10} K_{k, l}^{\mathrm{I}}-10 \gamma \log _{10}\left(\frac{d_{k, l}}{d_{0}}\right)-\psi_{\mathrm{dB}}\right)
\end{aligned}
$$

the wavelength of the transmitted wave by sensor $k$. Finally, reflected power via modulated backscattering by sensor $k$ at sink $m\left(P_{m, k}^{\mathrm{R}}\right)$ can be found as in (3). We use (3) for calculation of power gains of sensor nodes in distributed estimation distortion formulation in the next section.

\section{B. Distributed Estimation Scheme in WPSN}

The best linear unbiased estimator (BLUE) [8] is employed at sinks, due to fact that it does not require assumption of any specific distribution for sensed signal and noise. Observation $s_{k}(t)$ is the distorted version of random signal $\theta(t)$ by observation noise $n_{k}^{s}(t)$, i.e., $s_{k}(t)=\theta(t)+n_{k}^{s}(t)$, at sensor $k$ and time $t$. We also assume that both $\theta(t)$ and $n_{k}^{s}(t)$ are i.i.d. over time. Each sensor broadcast the signal $s_{k}(t)$ to sinks, where $\theta(t)$ is estimated from the received version of $s_{k}(t)$. We also assume that $\theta(t)$ and $n_{k}^{s}(t)$ have zero mean and has a power of $\sigma_{\theta}^{2}$ and $\sigma_{k}^{2}$, respectively. We assume sinks communicate with sensors over single hop via $k$ orthogonal channels that experience independent shadowing $(\psi)$ and zero-mean AWGN $\left(n_{k}^{c}\right)$. We also assume that synchronization is achieved between each sensor, RF source, and sink, and hence, effect of phase is eliminated. Motivated by the results in [9], each sensor employs analog amplify and forward uncoded transmission. Under independence over time assumption, time indices are ignored for all variables. Power gain $\delta_{k, m}$ for the sensed signal $s_{k}$ by sensor $k$ that is received at sink $m$ can be determined as

$$
\delta_{k, m}=\sqrt{\frac{P_{k, m}^{\mathrm{R}}}{\sigma_{\theta}^{2}+\sigma_{k}^{2}}}
$$

Received sample vector at sink $m$, i.e., $\mathbf{r}_{\mathbf{m}}$, is as $\mathbf{r}_{\mathbf{m}}=$ $\mathbf{h}_{\mathbf{m}} \theta+\mathbf{n}_{\mathbf{m}}$, where $\mathbf{r}_{\mathbf{m}}=\left[r_{1, m}, \cdots, r_{K, m}\right]^{T}, \mathbf{h}_{\mathbf{m}}=$ $\left[\delta_{1, m}, \cdots, \delta_{K, m}\right]^{T}$, and $\mathbf{n}_{\mathbf{m}}=\left[\delta_{1, m} n_{1}^{s}+n_{1}^{c}, \cdots, \delta_{K, m} n_{K}^{s}+\right.$ $\left.n_{K}^{c}\right]^{T}$. Noise covariance matrix $\mathbf{R}_{\mathbf{m}}$ for received samples at sink $m$ is a $K$ dimensional rectangular diagonal matrix whose diagonal entries are $\delta_{k, m}^{2} \sigma_{k}^{2}+\eta_{k}^{2}$ for $k=1 \cdots K$, and other entries are 0 . Hence, the mean square error for BLUE can be determined as [8]

$$
\begin{aligned}
\operatorname{Var}\left[\hat{\theta}_{m}\right] & =\left[\mathbf{h}_{\mathbf{m}}{ }^{T} \mathbf{R}_{\mathbf{m}}{ }^{-1} \mathbf{h}_{\mathbf{m}}\right]^{-1} \\
& =\left(\sum_{k=1}^{K} \frac{1}{\sigma_{k}^{2}+\eta_{k}^{2} / \delta_{k, m}^{2}}\right)^{-1}
\end{aligned}
$$

It is deduced from (5) that the estimation distortion decreases with increasing $\delta_{k, m}$. Distortion in the event estimation indicates how reliably event features can be extracted from sensor observations. In Section IV, we analyse the distortion function to quantitatively explore the effects of RF source and estimation diversity in PODS.

\section{Instantaneous Throughput and Cognitive Cycle}

Periodic spectrum sensing durations $\left(\tau_{s}^{c}\right)$ and spectrum handoffs following licensed user detection brought by OSA impose interruptions on event reporting. Here, we derive instantaneous throughput $T_{i}^{c}$ for event reporting in PODS in opportunistically accessed channel $c$. We assume that licensed user communication in each channel follows an i.i.d. ON/OFF random process [10], [11], [12], [13].

We define average instantaneous throughput in channel $c$ as the ratio of average duration spent for communication to average duration spent in that channel, i.e., $\bar{T}_{i}^{c}=\frac{\bar{\tau}_{d}^{c}}{\bar{\tau}^{c}}$. In order to find $\frac{\bar{\tau}_{d}^{c}}{\bar{\tau}^{c}}$ ratio, firstly, we determine $\bar{\tau}_{c}$, i.e., average duration from starting communication in channel $c$ to moving to a new vacant channel with completed spectrum handoff, as follows

$$
\bar{\tau}_{c}=\bar{\tau}_{h}^{c}+\bar{I}^{c}\left(\tau_{s}^{c}+\tau_{t}^{c}\right)
$$

where $\bar{\tau}_{h}^{c}$ is mean spectrum handoff duration, $\bar{I}^{c}$ is mean number of $\left(\tau_{s}^{c}+\tau_{t}^{c}\right)$ durations before licensed user arrives.

To calculate $\bar{I}^{c}$, we define the probability of having $i$ intervals in a channel $c$ as

$$
P\left[I^{c}=i\right]=\left(1-P_{\mathrm{ON}}^{c}\right)^{i-1} P_{\mathrm{ON}}^{c}
$$

where $P_{\mathrm{ON}}^{c}$ is licensed user $\mathrm{ON}$ probability in working channel. Mean of $I^{c}$ can be found as

$$
\bar{I}^{c}=\sum_{i=1}^{\infty} i \cdot P\left[I^{c}=i\right]=\frac{1}{P_{\mathrm{ON}}^{c}} .
$$

To find $\bar{\tau}_{h}^{c}$, we find conditional probability of finding a vacant channel via $l$ consecutive spectrum handoffs given that a licensed user is communicating at the current channel $\left(P\left[L^{c}=l \mid P_{\mathrm{ON}}^{0}\right]\right)$ as

$$
P\left[L^{c}=l \mid P_{\mathrm{ON}}^{0}\right]=\frac{P_{\mathrm{OFF}}^{l} \cdot \prod_{c=0}^{l-1}\left(1-P_{\mathrm{OFF}}^{c}\right)}{\left(1-P_{\mathrm{OFF}}^{0}\right)}
$$

where we assume licensed user arrivals at different channels are independent from each other. On the other hand, time consumed when performing channel switching and spectrum 
sensing for licensed activity detection while seeking a vacant channel through $l$ consecutive handoff can be found as

$$
\tau_{h}^{c}(l)=\sum_{m=0}^{l-1}\left(\tau_{\mathrm{cs}}^{m, m+1}+\tau_{s}^{m+1}\right)
$$

where $\tau_{c s}^{m, m+1}$ is channel switching time when moving from channel $m$ to $m+1$. As a result, $\bar{\tau}_{h}^{c}$ can be obtained as

$$
\begin{aligned}
\bar{\tau}_{h}^{c}= & \sum_{l=1}^{C} P\left(L^{c}=l \mid P_{\mathrm{ON}}^{0}\right) \\
& \cdot \sum_{k=0}^{\infty}\left(k \cdot \tau_{h}^{c}(C)+\tau_{h}^{c}(l)\right) P\left(L^{c}=C+\right)^{k}
\end{aligned}
$$

where $P\left(L^{c}=C+\right)$ is the probability of not finding a vacant channel after traversing channel list and returning to initial channel, which is given by $\prod_{c=1}^{C}\left(1-P_{\mathrm{OFF}}^{c}\right)$.

Only in $\tau_{t}^{c}$ durations sensors can deliver sensed phenomenon, since while spectrum sensing communication cannot be performed at the same time. Then, mean of total data transmission duration in a channel $c\left(\bar{\tau}_{d}^{c}\right)$ is found as

$$
\bar{\tau}_{d}^{c}=\bar{I}^{c} \cdot \tau_{t}^{c}
$$

Finally, mean of instantaneous throughput in channel $c$ can be found by dividing $\bar{\tau}_{d}^{c}$ with $\bar{\tau}_{c}$ as

$$
\begin{aligned}
\bar{T}_{i}^{c} & =\frac{\bar{\tau}_{d}^{c}}{\bar{\tau}_{c}} \\
& =\frac{\frac{\tau_{t}^{c}}{P_{\mathrm{ON}}^{c}}}{\bar{\tau}_{h}^{c}+\frac{1}{P_{\mathrm{ON}}^{c}}\left(\tau_{s}^{c}+\tau_{t}^{c}\right)}
\end{aligned}
$$

Formulated mean instantaneous throughput $\bar{T}_{i}^{c}$ is used to incorporate effect of OSA, i.e., silent spectrum sensing and handoff periods, into our estimation distortion and energy efficiency performance analysis.

\section{Energy-efficient Power Control In PODS}

Here, we give details of energy-efficient power level adjustment of RF sources in PODS. We formulate the energy consumption minimization problem as follows

$$
\begin{gathered}
\min \sum_{l} P_{l}^{\mathrm{RF}} \\
D \leq D_{o}
\end{gathered}
$$

where $D$ is calculated as in (5). In order to satisfy event estimation distortion requirements, sufficient number of samples affected by minimum amount of noise should be received at sink. According to the highest noise experiencing node, new power level can be set for RF power sources. Since power level will be set according lowest signal-to-noise ratio (SNR) having sensor node, reports of other sensor nodes

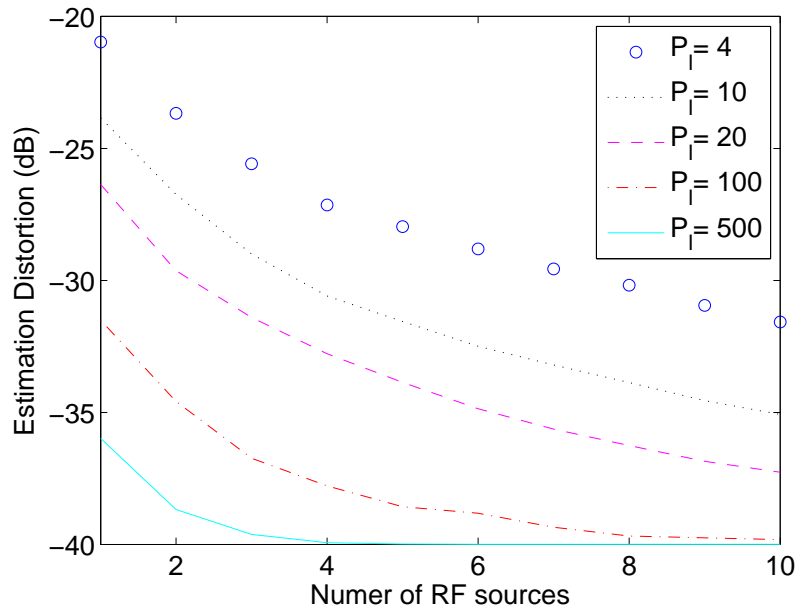

Figure 3. Estimation distortion vs number of RF sources and their power levels in PODS.

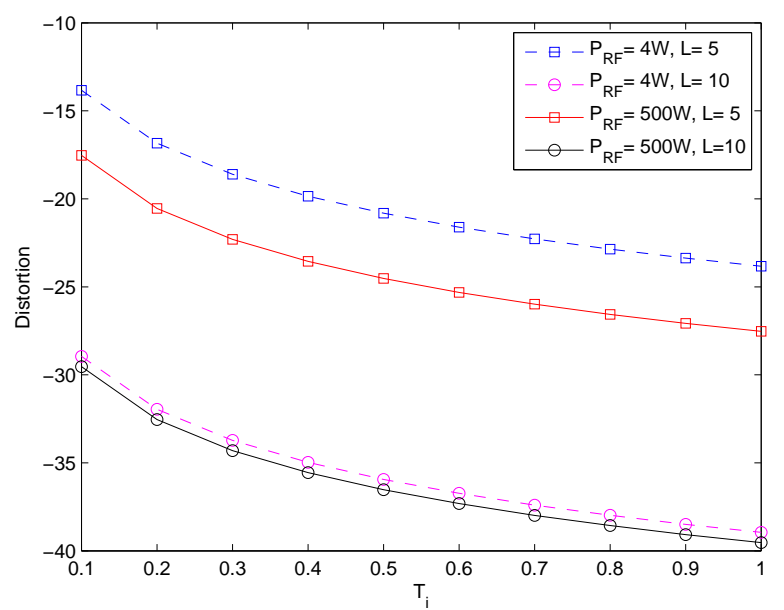

Figure 4. Estimation distortion vs instantaneous throughput considering specifically effect of opportunistic spectrum access.

will already have sufficiently large SNR. Desired power gain for lowest SNR having sensor node $k$ to satisfy distortion constraint $D_{o}$ at sink $m$ is founded as

$$
\delta_{k, m}^{2}=\frac{D_{o} \eta_{k}^{2}}{K \cdot \tau_{e} \cdot f_{s} \cdot \bar{T}_{i}^{c}-D_{o} \sigma_{k}^{2}}
$$

where $K$ is the number of sensors, $\tau_{e}$ is the estimation interval at the end of which event is estimated from collected samples from passive sensors, and $f_{s}$ is the sampling rate of passive sensors.

We control $P_{l}^{\mathrm{RF}}$ according to the lowest power gain of sensor nodes, such that lowest power gain having sensor will satisfy to have desired power gain $\delta_{k, m}^{2}$ to achieve required estimation distortion $D_{o}$ at sink, and other passive sensors' 


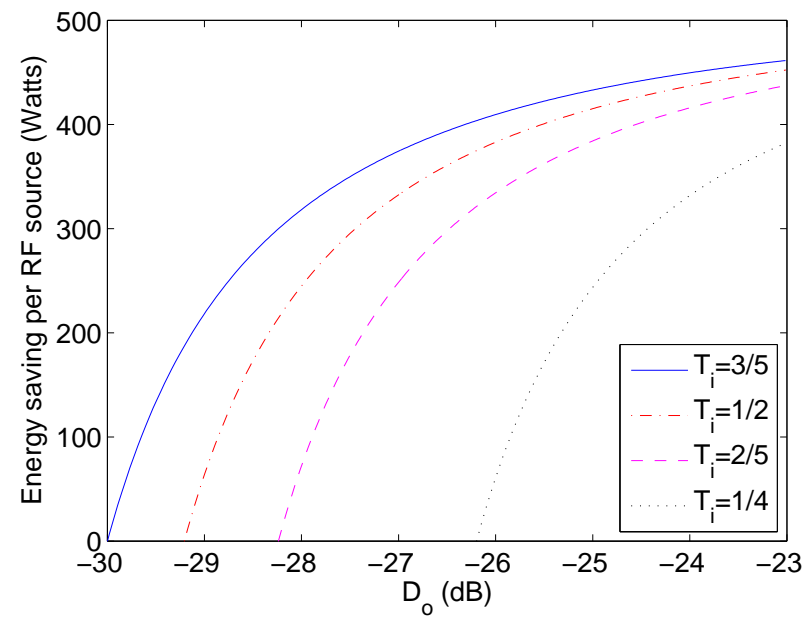

Figure 5. Estimation distortion vs number of RF sources and their power levels in PODS, $\eta^{2}=10^{-5}$.

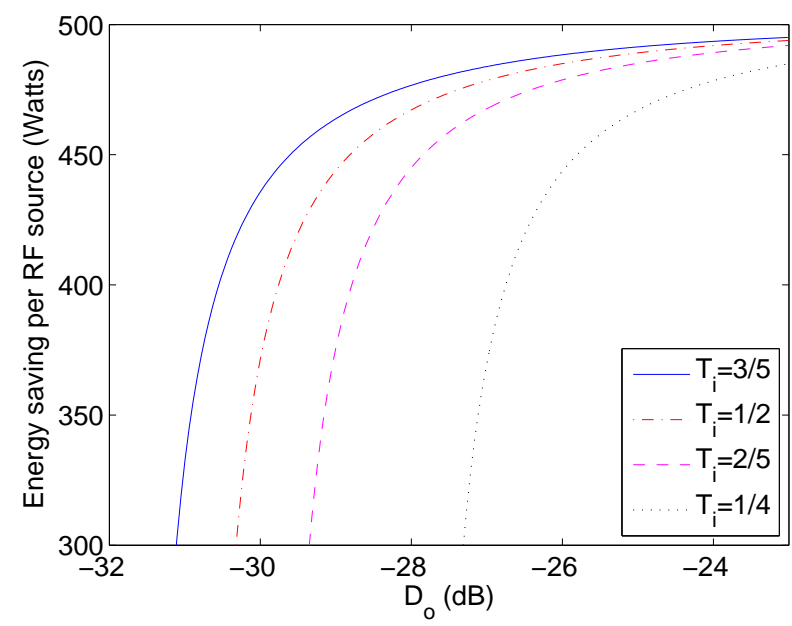

Figure 6. Estimation distortion vs number of RF sources and their power levels in PODS, $\eta^{2}=10^{-6}$.

power gain will be guaranteed to be greater than the desired one for lowest SNR having sensor node.

\section{Performance Evaluation}

For performance evaluation, 100 passive sensor nodes are placed in a 50x50 m area with a grid topology. For RF power source diversity investigation, number of randomly placed RF sources are varied from 1 to 20 inside this field, and a sink is placed at the center of the grid. For estimation diversity analysis in PODS, $P_{l}^{\mathrm{RF}}$ is kept constant for all $l$, and varied from 4 to $500 \mathrm{~W}$. $\gamma$ and $\psi_{\mathrm{dB}}$ are kept constant as 2.5 and 1 , respectively. $d_{0}$ is taken as $100 \mathrm{~m}$. At least $10 \mathrm{~mW}$ must be intercepted by sensor node to operate [2], and received signal power level at RF sources

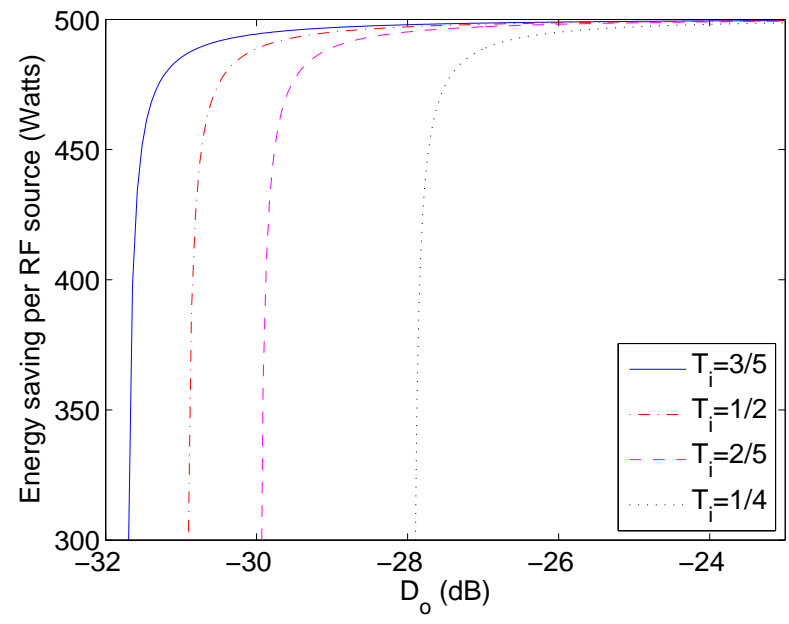

Figure 7. Estimation distortion vs number of RF sources and their power levels in PODS, $\eta^{2}=10^{-7}$.

must be greater than $-90 \mathrm{dBm}$ due to typical receiver sensitivity levels [7]. $\sigma_{\theta}^{2}$ and $\sigma_{k}^{2}$ are assumed to be 1 and 0.01 , respectively. Antenna gains $G_{l}^{\mathrm{RF}}$ are taken to be unity for all RF sources. AWGN variance $\left(\eta_{k}^{2}\right)$ is set to $-90 \mathrm{dBm}$ for all $k$. We repeated simulations 200 times and averaged obtained results. Simulation results for RF power source diversity are presented in Fig. 3.

In Fig. 3, effect of RF power source levels and number on estimation distortion is shown. For a constant power level, while we increase number of RF sources, estimation distortion decreases with increasing number of reporting sensors. This is due to fact that the number of activated sensor nodes increases with the number of RF sources. Furthermore, when power level of RF sources is increased, in Fig. 3 it is seen that estimation distortion decreases for a fixed number of RF sources deployed in field. This is due to fact that as the power level of RF sources increases, power gain of active sensors and number of activated sensors also increase. With the incorporation of various sensors' reports (increase of number of reporting sensors) and increasing their reports' SNR (increase of power gain for sensor-tosink path) estimation diversity is obtained, and this provides reduction in estimation distortion for achievement of reliable opportunistic distributed sensing in WPSN.

Achieved event estimation distortion cannot be further reduced after a certain number of RF sources or power level as can be seen in Fig. 3, respectively. This reveals that joint consideration of the required number of RF sources and power level for reliable event estimation can yield significant energy savings. Furthermore, it is observed that increasing the number of RF sources improves event estimation performance more effectively than increase in the power level of RF sources. On the other hand, in order to achieve reliability, 
higher power radiation by RF sources may be required based on channel characteristics, since higher number of reporting source nodes may result in other problems as higher contention delays when orthogonal channels cannot be reserved for each passive sensor. For reliable event estimation, desired number of RF sources and their power level is highly depending on channel characteristics and density of sensor nodes. Therefore, number of RF source occupied in the field and their power levels must be adjusted accordingly.

As well as RF source diversity, simulation results are presented for effect of instantaneous throughput reduction due to opportunistic spectrum access in Fig. 4. For different number of RF sources and their power levels achieved distortion is revealed. Furthermore, proposed energy-efficient power level adjustment method's energy saving performance is assessed as well. Simulation results regarding energy consumption minimization in PODS is presented in Fig. 5, 6 , and 7 for various channel noise levels. These results show the relevancy of the achieved energy savings and the channel noise for different instantaneous throughput values. It is seen that for lower channel noise values it is possible to save energy for higher estimation distortion requirements when compared to higher channel nose case. When $\eta^{2}=10^{-5}$ case is compared to $\eta^{2}=10^{-7}$ case in Fig. 5 and Fig. 7, respectively, saved energy difference is greater about $100 \mathrm{~W}$ for $D_{0}=-25$. As the distortion requirement gets higher, savable energy becomes close to each other for different channel noise values.

\section{CONCLUSiON}

In this paper, we study energy-efficient RF source power control for opportunistic distributed sensing in wireless passive sensor networks (WPSN). First, an analysis of reflected power at sink from sensor nodes and instantaneous throughput due to opportunistic access is performed. Then, event estimation distortion is modelled with respect to number and power level of RF sources, and available spectrum opportunities. We provide an energy-efficient RF source power level control algorithm incorporating formulated wireless power transfer and instantaneous throughput in passive opportunistic distributed sensing (PODS). Simulation experiments reveal that via controlling number and power level of RF sources, significant energy savings can be achieved while desired estimation distortion is reached. Future work includes development of efficient RF source deployment and non-equal power adjustment algorithms to improve distributed sensing performance, as well as mac protocol design and physical layer performance analysis for energy-efficient communication in PODS.

\section{ACKNOWLEDGMENTS}

This work was supported in part by the Turkish Scientific and Technical Research Council under grant \#110E249, by the Turkish National Academy of Sciences Distinguished Young Scientist Award Program (TUBA-GEBIP), and by the TURK TELEKOM under Grant Number 11315-04.

\section{REFERENCES}

[1] O. B. Akan, M. T. Isik, and B. Baykal, "Wireless Passive Sensor Networks," IEEE Communications Magazine, vol. 47, no. 8, pp. 92-99, August 2009.

[2] F. Kocer, P. M. Walsh, and M. P. Flynn, "Wireless, remotely powered telemetry in $0.25 \mathrm{~m}$ CMOS," in Proc. IEEE Radio Frequency Integrated Circuits Symposium (RFIC), pp. 339342, June 2004.

[3] M. Kossel, H. R. Benedickter, R. Peter, and W. Bachtold, "Microwave backscatter modulation systems," 2000 IEEE MTT-S Digest, vol. 3, pp. 1427-1430, June 2000.

[4] M. Zuniga and B. Krishnamachari, "Analyzing the transitional region in low power wireless links," in Proc. IEEE SECON'04, Oct. 2004, pp. 517-526.

[5] A. O. Bicen, and O. B. Akan, "RF Power Source and Estimation Diversity in Distributed Sensing with Passive Wireless Communications," in Proc. 30th URSI GASS 2011, August 2011.

[6] I. F. Akyildiz, W. Y. Lee, M. C. Vuran, and S. Mohanty, "NeXt Generation/Dynamic Spectrum Access/Cognitive Radio Wireless Networks: A Survey," Comput. Netw. J., vol. 50, no. 13, pp. 2127-2159, 2006.

[7] C. A. Balanis, Antenna Theory: Analysis and Design, 2nd ed. John Wiley and Sons Inc., 1997.

[8] J. M. Mendel, Lessons in Estimation Theory for Signal Processing, Communications, and Control, Englewood Cliffs, NJ: Prentice-Hall, 1995.

[9] M. Gastpar, B. Rimoldi, and M. Vetterli, "To code, or not to code: lossy source-channel communication revisited," IEEE Trans. Inf. Theory, vol. 49, pp. 1147-1158, May 2003.

[10] K. Sriram and W. Whitt, "Characterizing superposition arrival processes in packet multiplexer for voice and data," IEEE $J$. Sel. Areas Commun., vol. 4, no. 6, pp. 833846, Sep. 1986.

[11] R. Urgaonkar and M. J. Neely, "Opportunistic scheduling with reliability guarantees in cognitive radio network," in Proc. IEEE INFOCOM 2008, 2008.

[12] S. Huang, X. Liu, and Z. Ding, "Opportunistic spectrum access in cognitive radio networks," in Proc. IEEE INFOCOM 2008, pp. 1734-1743, 2008.

[13] W. Y. Lee, and I. F. Akyildiz, "Optimal Spectrum Sensing Framework for Cognitive Radio Networks," IEEE Trans. on Wireless Commun., vol. 7, no. 10, pp. 3845-3857, 2008. 